Article

\title{
Integrated Metabolomic and Transcriptomic Analysis Reveals Differential Mechanism of Flavonoid Biosynthesis in Two Cultivars of Angelica sinensis
}

\author{
Tiantian Zhu ${ }^{1,2}{ }^{\text {, Minghui Zhang }}{ }^{1}$, Hongyan $\mathrm{Su}^{3}$, Meiling $\mathrm{Li}^{3}$, Yuanyuan Wang ${ }^{1,2}$, Ling Jin ${ }^{1,2, *}$ and \\ Mengfei Li ${ }^{3, *(\mathbb{D})}$ \\ 1 College of Pharmacy, Gansu University of Chinese Medicine, Lanzhou 730101, China; \\ ztt0935@gszy.edu.cn (T.Z.); zmh0819@tom.com (M.Z.); wyy19880821@126.com (Y.W.) \\ 2 Northwest Collaborative Innovation Center for Traditional Chinese Medicine, Lanzhou 730000, China \\ 3 State Key Laboratory of Aridland Crop Science, Gansu Agricultural University, Lanzhou 730070, China; \\ Shy922322@163.com (H.S.); mlli1996@163.com (M.L.) \\ * Correspondence: jinl@gszy.edu.cn (L.J.); lmf@gsau.edu.cn (M.L.)
}

check for updates

Citation: Zhu, T.; Zhang, M.; Su, H.; Li, M.; Wang, Y.; Jin, L.; Li, M. Integrated Metabolomic and Transcriptomic Analysis Reveals Differential Mechanism of Flavonoid Biosynthesis in Two Cultivars of Angelica sinensis. Molecules 2022, 27, 306. https://doi.org/10.3390/ molecules27010306

Academic Editors: Giuseppe Dionisio and Vittorio Calabrese

Received: 28 November 2021

Accepted: 2 January 2022

Published: 4 January 2022

Publisher's Note: MDPI stays neutral with regard to jurisdictional claims in published maps and institutional affiliations.

Copyright: (C) 2022 by the authors. Licensee MDPI, Basel, Switzerland. This article is an open access article distributed under the terms and conditions of the Creative Commons Attribution (CC BY) license (https:// creativecommons.org/licenses/by/ $4.0 /)$.

\begin{abstract}
Angelica sinensis is a traditional Chinese medicinal plant that has been primarily used as a blood tonic. It largely relies on its bioactive metabolites, which include ferulic acid, volatile oils, polysaccharides and flavonoids. In order to improve the yield and quality of $A$. sinensis, the two cultivars Mingui 1 (M1), with a purple stem, and Mingui 2 (M2), with a green stem, have been selected in the field. Although a higher root yield and ferulic acid content in M1 than M2 has been observed, the differences of flavonoid biosynthesis and stem-color formation are still limited. In this study, the contents of flavonoids and anthocyanins were determined by spectrophotometer, the differences of flavonoids and transcripts in M1 and M2 were conducted by metabolomic and transcriptomic analysis, and the expression level of candidate genes was validated by qRT-PCR. The results showed that the contents of flavonoids and anthocyanins were 1.5- and 2.6-fold greater in M1 than M2, respectively. A total of 26 differentially accumulated flavonoids (DAFs) with 19 up-regulated (UR) and seven down-regulated (DR) were obtained from the 131 identified flavonoids (e.g., flavonols, flavonoid, isoflavones, and anthocyanins) in M1 vs. M2. A total 2210 differentially expressed genes (DEGs) were obtained from the 34,528 full-length isoforms in M1 vs. M2, and 29 DEGs with 24 UR and 5 DR were identified to be involved in flavonoid biosynthesis, with 25 genes (e.g., CHS1, CHI3, F3H, DFR, ANS, CYPS and UGTs) mapped on the flavonoid biosynthetic pathway and four genes (e.g., RL1, RL6, MYB90 and MYB114) belonging to transcription factors. The differential accumulation level of flavonoids is coherent with the expression level of candidate genes. Finally, the network of DAFs regulated by DEGs was proposed. These findings will provide references for flavonoid production and cultivars selection of $A$. sinensis.
\end{abstract}

Keywords: Angelica sinensis; cultivar; flavonoids; anthocyanins; metabolomics; transcriptomics

\section{Introduction}

Angelica sinensis (Oliv.) Diels (syn. Angelica polymorpha Maxim. var. sinensis Oliv.) is an Apiaceae (alt. Umbelliferae) perennial rhizomatous species and commonly named as Dang gui, Dong quai and Toki [1]. The species is originally native to China, with a population center in Gansu and widely cultivated at altitudes of 2000 to $3000 \mathrm{~m} \mathrm{[1-3].} \mathrm{The} \mathrm{roots}$ were first recorded in the earliest known herbal text "Shen Nong Ben Cao Jing", and have been used as a traditional Chinese medicine for nourishing the blood, regulating female menstrual disorders, relieving pain, and relaxing the bowels, etc., for over 2000 years [4,5]. Recently, the roots have been used as potential treatments of acute ischemic stroke, chronic obstructive pulmonary disease with pulmonary hypertension, as well as for its cardiocerebrovascular, immunomodulatory and antioxidant effects [5-7]. Phytochemical and 
pharmacological investigations have demonstrated that these therapeutic properties largely rely on bioactive metabolites including ferulic acid, volatile oils, polysaccharides and flavonoids $[1,5,8]$.

In order to improve the yield and quality of $A$. sinensis, strategies undertaken include selecting suitable cultivation areas [3,9], cultivating with standard methods [10-12], inhibiting early bolting and flowering [13-15], and breeding fine cultivars [16]. Currently, six A. sinensis cultivars that are recorded include: Mingui 1 (M1) with a purple stem, Mingui 2 (M2) with a green stem, and Mingui 5, with bigger leaves selected using a systematic breeding method, as well as Mingui 3, Mingui 4 and 6 from the M1 irradiated by heavy ion $\left(55 \mathrm{Mev} / \mathrm{u}^{40} \mathrm{Ar}^{17+}\right)$ [17]. Among the six cultivars, the M1 dominates in the production with cultivated area at over 41,000 ha $(95 \%)$. Due to its high yield, the M2 is gradually accepted due to its low rate of early bolting and flowering [16-18].

For the difference of bioactive metabolites between M1 and M2, greater ferulic acid content and less ligustilide content has been observed in M1 in comparison with M2 [19,20]. The differences of other bioactive metabolites, including volatile oils, polysaccharides and flavonoids, as well as the stem-color formation between M1 and M2, have not been investigated. In this study, we examined the differences of flavonoids and transcripts based on metabolomic and transcriptomic analysis, and found that the flavonoid and anthocyanin contents were greater in M1 than M2; 26 flavonoids were differentially accumulated; and 29 genes involved in flavonoid biosynthesis were differentially expressed in M1 vs. M2.

\section{Results}

\subsection{Differenence of Flavonoid and Anthocyanin Contents between the Two Cultivars}

As is shown in Figure 1, a significant difference of flavonoid and anthocyanin contents was observed, with a 1.48- and 2.57-fold greater amount in M1 than M2, respectively.
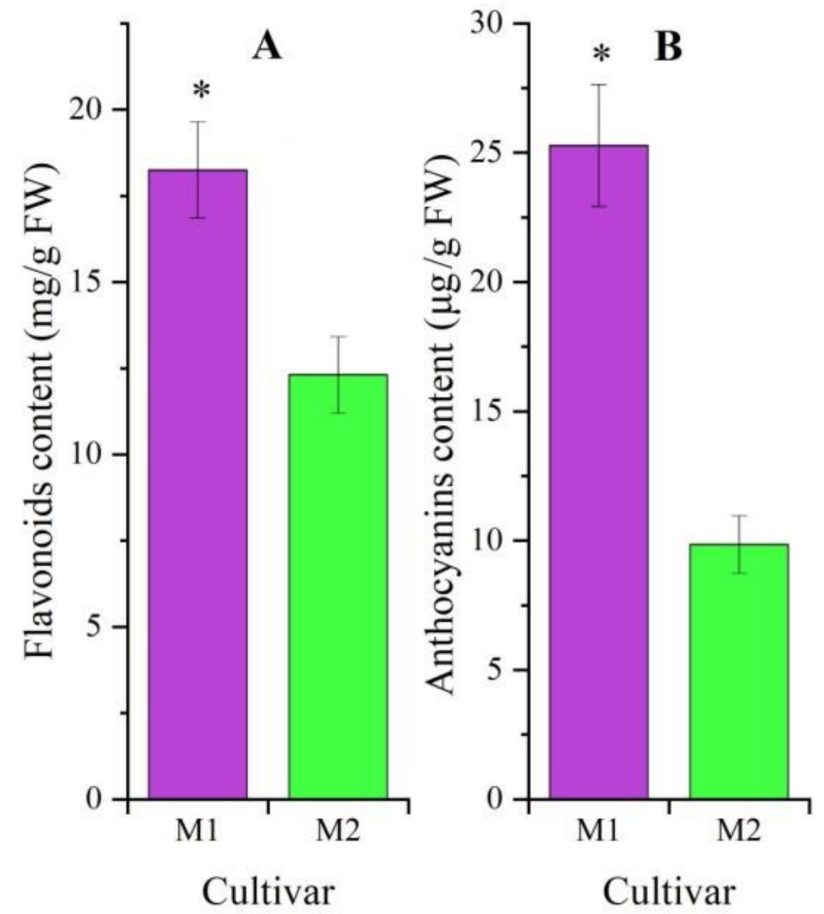

Figure 1. Contents of flavonoids (A) and anthocyanins (B) in Mingui 1 (M1) and Mingui 2 (M2) (mean $\pm \mathrm{SD}, n=3$ ). A $t$-test was performed for independent treatments, and the "** is considered significant at $p<0.05$ between M1 and M2. 


\subsection{Targeted-Flavonoids Metabolomic Analysis}

2.2.1. Identification of Differentially Accumulated Flavonoids (DAFs) in M1 vs. M2

A total of 131 flavonoids that were identified by LC-ESI-MS/MS analysis include flavonols (51), flavonoid (36), flavanols (8), chalcones (7), dihydroflavone (7), dihydroflavonol (7), isoflavones (6), anthocyanins (5), and flavonoid carbonoside (4) (Figure 2). Among them, 26 (19 UR and 7 DR) flavonoids that were differentially accumulated in M1 vs. M2 based on the principal component analysis (PCA) and orthogonal projection to latent structures-discriminant analysis (OPLS-DA) (Figures S1 and S2). The specific flavonoids and their differential accumulation levels in M1 vs. M2 are shown in Table 1 and Figure S3.

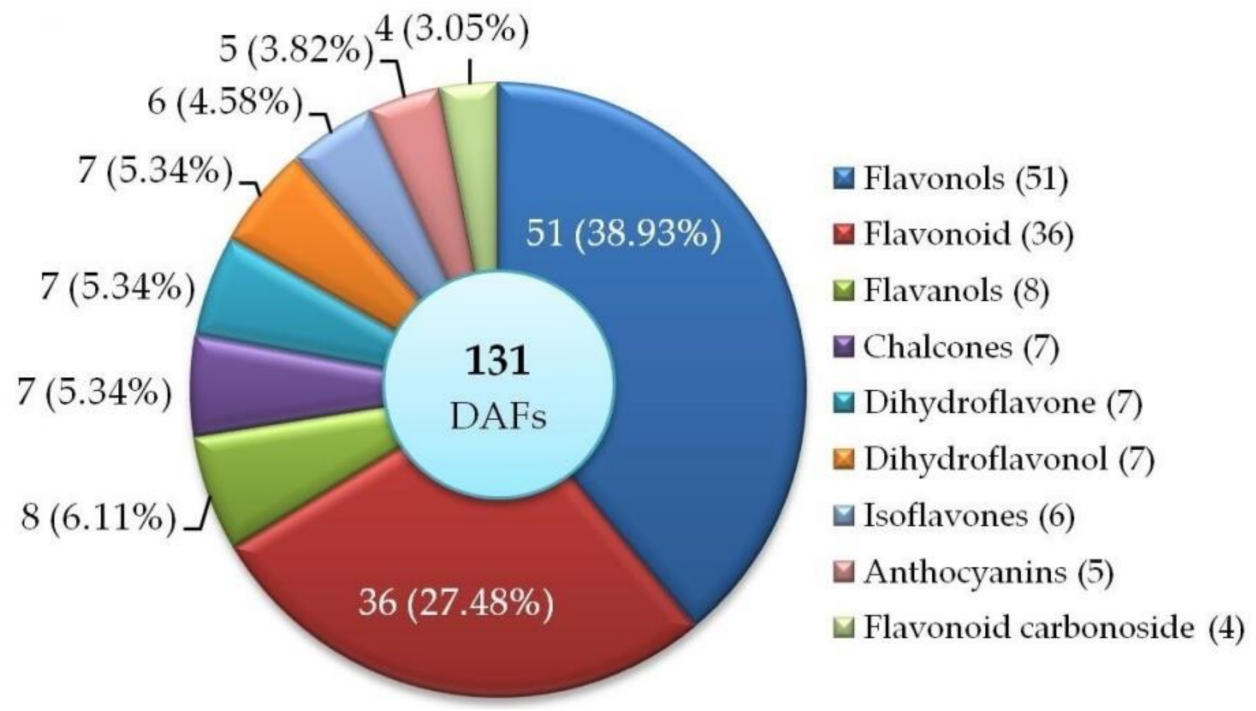

Figure 2. Distribution and classification of DAFs in M1 vs. M2.

Table 1. Classification of DAFs and their differential accumulation levels in M1 vs. M2 (mean \pm SD, $n=3)$.

\begin{tabular}{lllc}
\hline No. & Compounds Name & Formula & $\log _{2}$ FC(M1 vs. M2) \\
\hline 1 & Quercetin-3-O-(6'-O-arabinosyl) glucoside & $\mathrm{C}_{26} \mathrm{H}_{28} \mathrm{O}_{16}$ & $0.90 \pm 0.12$ \\
2 & Quercetin-3-O-arabinoside (Guaijaverin) & $\mathrm{C}_{20} \mathrm{H}_{18} \mathrm{O}_{11}$ & $0.65 \pm 0.07$ \\
3 & Quercetin-3-O-apiosyl(1 $\rightarrow$ 2) galactoside & $\mathrm{C}_{26} \mathrm{H}_{28} \mathrm{O}_{16}$ & $0.65 \pm 0.06$ \\
4 & Quercetin-3-O-sambubioside & $\mathrm{C}_{26} \mathrm{H}_{28} \mathrm{O}_{16}$ & $0.55 \pm 0.01$ \\
5 & Quercetin-3-O-xyloside (Reynoutrin) & $\mathrm{C}_{20} \mathrm{H}_{18} \mathrm{O}_{11}$ & $0.35 \pm 0.07$ \\
6 & Quercetin-3-O-rhamnoside (Quercitrin) & $\mathrm{C}_{21} \mathrm{H}_{20} \mathrm{O}_{11}$ & $0.34 \pm 0.06$ \\
7 & Quercetin-7-O-rutinoside & $\mathrm{C}_{27} \mathrm{H}_{30} \mathrm{O}_{16}$ & $0.21 \pm 0.02$ \\
8 & Quercetin-4'-O-glucoside (Spiraeoside) & $\mathrm{C}_{21} \mathrm{H}_{20} \mathrm{O}_{12}$ & $-1.02 \pm 0.11$ \\
9 & Quercetin-3-O-galactoside (Hyperin) & $\mathrm{C}_{21} \mathrm{H}_{20} \mathrm{O}_{12}$ & $-0.93 \pm 0.06$ \\
10 & Quercetin-3-O-glucoside (Isoquercitrin) & $\mathrm{C}_{21} \mathrm{H}_{20} \mathrm{O}_{12}$ & $-0.78 \pm 0.06$ \\
11 & Quercetin-7-O-glucoside & $\mathrm{C}_{21} \mathrm{H}_{20} \mathrm{O}_{12}$ & $-0.66 \pm 0.14$ \\
12 & Isorhamnetin-3-O-sophoroside & $\mathrm{C}_{28} \mathrm{H}_{32} \mathrm{O}_{17}$ & $0.63 \pm 0.07$ \\
13 & Isorhamnetin-3-O-Glucoside & $\mathrm{C}_{22} \mathrm{H}_{22} \mathrm{O}_{12}$ & $0.38 \pm 0.01$ \\
14 & Rhamnetin-3-O-Glucoside & $\mathrm{C}_{22} \mathrm{H}_{22} \mathrm{O}_{12}$ & $0.38 \pm 0.01$ \\
15 & Chrysoeriol-5-O-glucoside & $\mathrm{C}_{22} \mathrm{H}_{22} \mathrm{O}_{11}$ & $-2.17 \pm 0.21$ \\
16 & Naringenin-7-O-glucoside (Prunin) & $\mathrm{C}_{21} \mathrm{H}_{22} \mathrm{O}_{10}$ & $1.15 \pm 0.16$ \\
17 & Hesperetin-5-O-glucoside & $\mathrm{C}_{22} \mathrm{H}_{24} \mathrm{O}_{11}$ & $-0.86 \pm 0.11$ \\
18 & 6-Hydroxykaempferol-7,6-O-Diglucoside & $\mathrm{C}_{27} \mathrm{H}_{30} \mathrm{O}_{17}$ & $0.47 \pm 0.06$ \\
19 & Kaempferol-4'-O-glucoside & $\mathrm{C}_{21} \mathrm{H}_{20} \mathrm{O}_{11}$ & $-0.39 \pm 0.05$ \\
\hline
\end{tabular}


Table 1. Cont.

\begin{tabular}{lllc}
\hline No. & Compounds Name & Formula & $\log _{2}$ FC(M1 vs. M2) \\
\hline 20 & Isosalipurposide (Phlorizin Chalcone) & $\mathrm{C}_{21} \mathrm{H}_{22} \mathrm{O}_{10}$ & $1.56 \pm 0.24$ \\
21 & Butin-7-O-glucoside & $\mathrm{C}_{21} \mathrm{H}_{22} \mathrm{O}_{10}$ & $0.95 \pm 0.21$ \\
22 & Luteolin-7-O-rutinoside & $\mathrm{C}_{27} \mathrm{H}_{30} \mathrm{O}_{15}$ & $0.37 \pm 0.06$ \\
23 & Pelargonidin-3-O-glucoside-5-O-arabinoside & $\mathrm{C}_{26} \mathrm{H}_{29} \mathrm{O}_{14}+$ & $18.66 \pm 1.22$ \\
24 & Cyanidin-3-O-glucoside (Kuromanin) & $\mathrm{C}_{21} \mathrm{H}_{21} \mathrm{O}_{11^{+}}$ & $19.15 \pm 1.73$ \\
25 & Cyanidin-3-O-sambubioside & $\mathrm{C}_{26} \mathrm{H}_{29} \mathrm{O}_{15}$ & $8.85 \pm 1.09$ \\
26 & Peonidin-3-O-sambubioside & $\mathrm{C}_{27} \mathrm{H}_{31} \mathrm{O}_{15}$ & $6.26 \pm 0.85$ \\
\hline
\end{tabular}

Note: The level of differential accumulation between M1 and M2 was determined with a criterion of variable importance in projection (VIP) $\geq 1$ and $t$-test $p \leq 0.05$.

\subsubsection{Pathway Enrichment of DAFs}

Among the 26 DAFs, seven metabolites were enriched in five pathways, including anthocyanin biosynthesis (cyanidin-3-O-glucoside and cyanidin-3-O-sambubioside, ko00942), flavone and flavonol biosynthesis (quercetin-3-O-rhamnoside, quercetin-3-O-glucoside and quercetin-3-O-sambubioside, ko00944), metabolic pathways (quercetin-3-O-glucoside, ko01100), flavonoid biosynthesis (naringenin-7-O-glucoside and isosalipurposide, ko00941), and biosynthesis of secondary metabolites (quercetin-3-O-glucoside, ko01110) based on the Kyoto Encyclopedia of Genes and Genomes (KEGG) databases analysis (Figure 3).

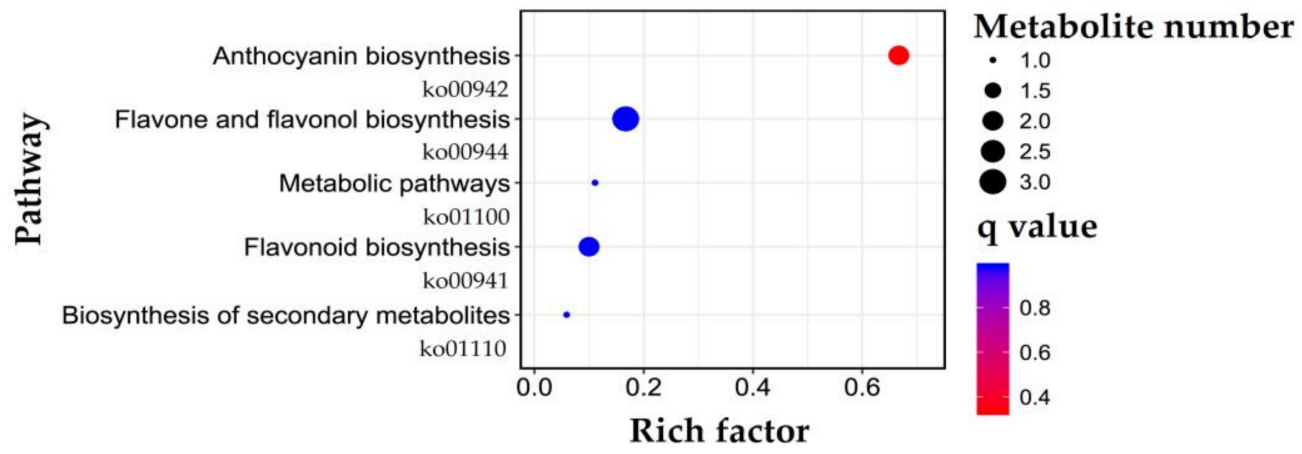

Figure 3. KEGG Orthology (KO) enrichment of the DAFs.

\subsection{Isoforms Analysis}

A total of 702,133 high-fidelity reads were extracted after 38 full passes of raw reads (Figure S4A), 45,026 polished high-quality isoforms were obtained using a Quiver calculation (Figure S4B), and 34,528 full-length isoforms were generated after the full-length non-chimeric (FLNC) reads clustered and integrated (Figure S4C).

The 34,528 isoforms were annotated against the KEGG $(33,241)$, KOG $(22,601), \mathrm{Nr}$ $(33,947)$ and SwissProt $(29,150)$ databases (Figure 4A), and the top 10 species distribution includes Daucus carota, Actinidia chinensis, Prunus dulcis, Camellia sinensis, Carica papaya, Angelica sinensis, Petroselinum crispum, Vitis vinifera, Brassica napus, and Artemisia annua (Figure 4B). 

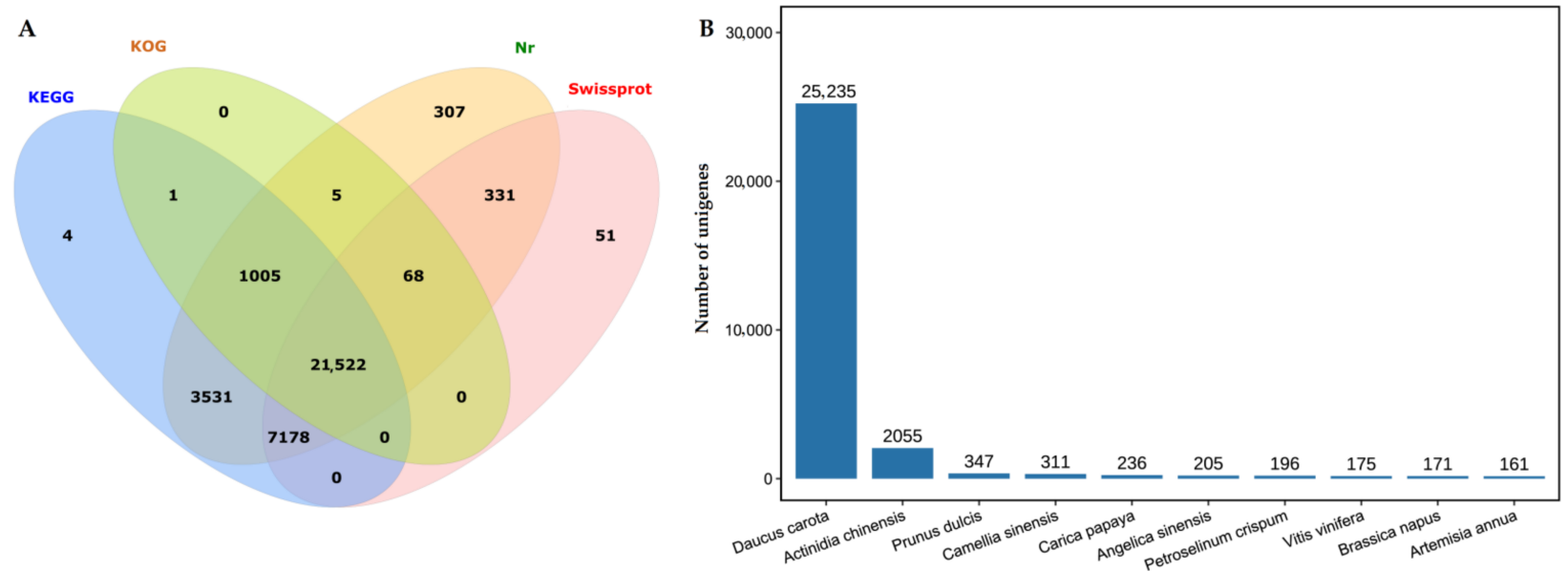

Figure 4. Basic annotation of the isoforms based on KEGG, KOG, Nr and SwissProt databases (A) and the top 10 species distribution against $\mathrm{Nr}(\mathbf{B})$.

\subsection{Transcriptomic Analysis between $M 1$ and $M 2$}

\subsubsection{Global Gene Analysis}

To reveal molecular mechanisms responsible for the difference of flavonoid accumulation and the stem-color formation, comparison of gene transcription between M1 and M2 was performed. After data filtering, 38.65 and 38.73 million high-quality reads were collected, and 27.92 and 28.36 multiple mapped reads were obtained from the M1 and M2, respectively. Meanwhile, the exon rate reached 100\% (Table 2).

Table 2. Summary of sequencing data of M1 and M2 (mean $\pm \mathrm{SD}, n=3$ ).

\begin{tabular}{lll}
\hline & M1 & M2 \\
\hline Filtered data & & $38.73 \pm 1.90$ \\
Data of reads number (million) & $38.65 \pm 1.34$ & $5784.44 \pm 2.83$ \\
Data of reads number × read length (million) & $5773.93 \pm 2.00$ & $98.09 \pm 0.21$ \\
Q20 $\%$ ) & $97.82 \pm 0.03$ & $94.05 \pm 0.54$ \\
Q30(\%) & $93.39 \pm 0.08$ & $6.25 \pm 0.29$ \\
Mapped data against full-length isoforms & & $28.36 \pm 1.35$ \\
Data of unique mapped reads (million) & $6.21 \pm 0.19$ & $89.37 \pm 0.17$ \\
Data of multiple mapped reads (million) & $27.92 \pm 0.87$ & 100 \\
Mapping ratio (\%) & $88.32 \pm 0.31$ & 100 \\
Exon rate (\%) & & \\
\hline
\end{tabular}

\subsubsection{Identification of Differentially Expressed Genes (DEGs)}

A total of 2210 DEGs were observed from the 34,528 full-length isoforms, with 1110 UR and 1100 DR in M1 vs. M2 (Figure 5A), based on the Reads Per kb per Million (RPKM) value (Figures S5 and S6), PCA (Figure S7) and Pearson correlation analysis (Figure S8). The cluster heat map of the 2,210 DEGs was shown in Figure 5B. 

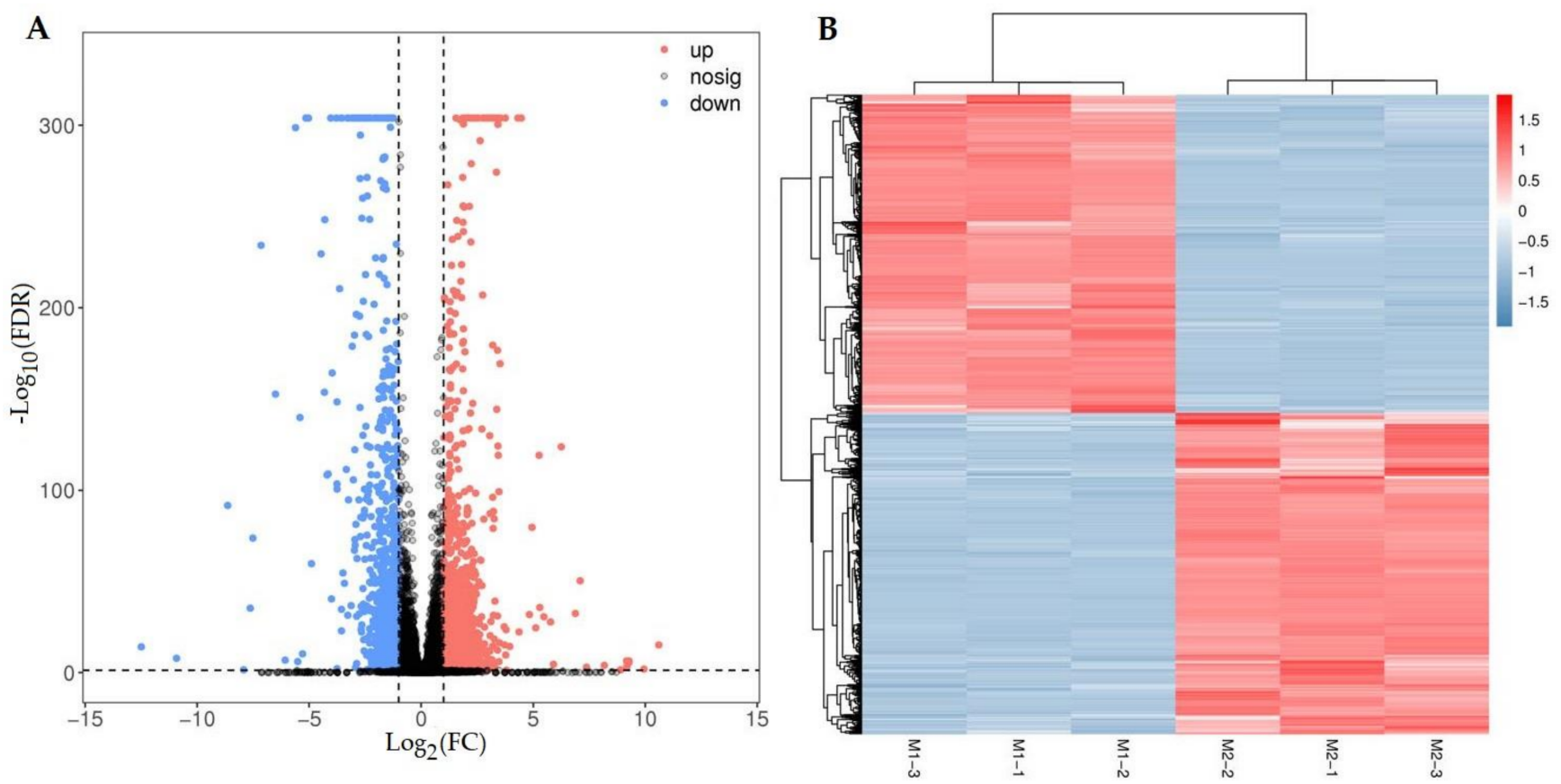

Figure 5. Volcano plot of differential comparison (A) and cluster heat map of the DEGs (B) in M1 vs. M2.

\subsubsection{Functional Annotation and Enrichment of DEGs}

The function of the 2210 DEGs was annotated against the Gene Ontology (GO) and KO databases. For the GO database, 48 terms were classified into biological process (22), cellular component (16), and molecular function (10) (Figure S9). For the KO database, 1784 DEGs were enriched 103 pathways, with top 10 pathways including: oxidative phosphorylation; metabolic pathways; linoleic acid metabolism; ABC transporters; alpha-Linolenic acid metabolism; nitrogen metabolism; phenylpropanoid biosynthesis; TCA cycle; cutin, suberine and wax biosynthesis; and pyruvate metabolism (Figure 6).

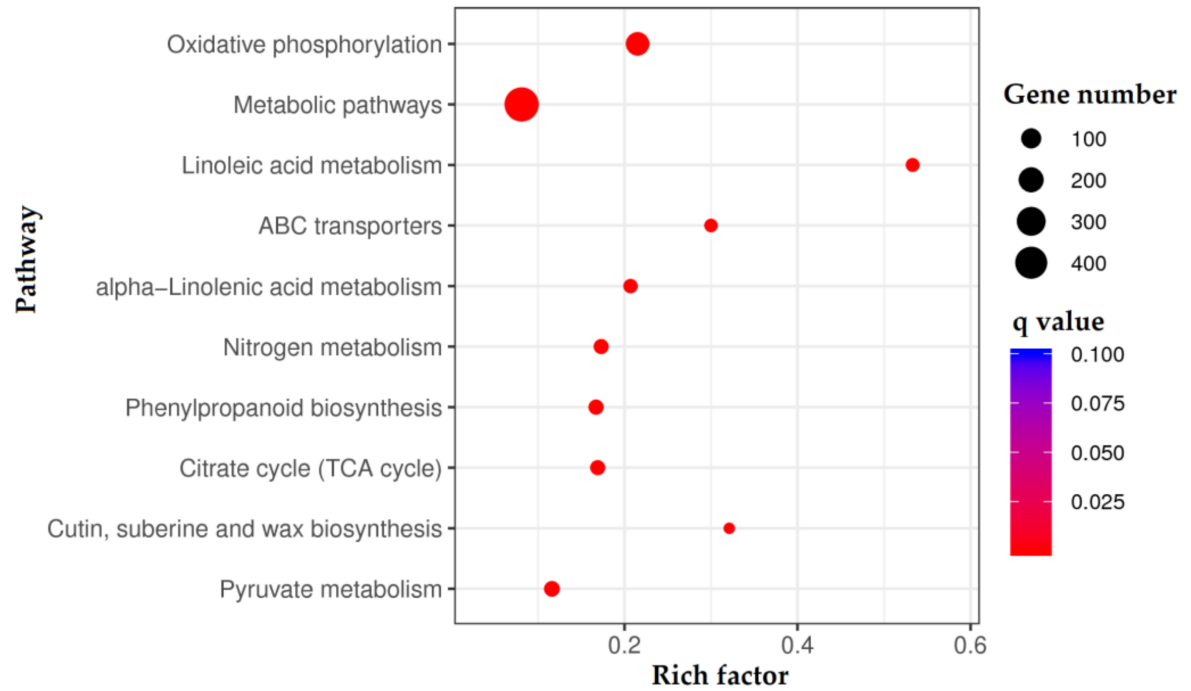

Figure 6. Top 10 pathways of KO enrichment of the DEGs.

\subsection{DEGs Involved in Flavonoids Biosynthesis}

Twenty-nine DEGs (24 UR and 5 DR) were identified to be involved in flavonoid biosynthesis. Twenty-five genes were mapped on flavonoid biosynthetic pathway with a 
1.04 to 8.63-fold UR for the 20 genes CHS1, CHI3, F3H, DFR, ANS, CGT, GT6, UGT85A8, F3GT1, P5MaT, CYP71A1, CYP71A9, CYP71D313, CYP71B26, CYP71B36, CYP72A219, CYP736A12, CYP76AD1, CYP76A2 and CYP77A3, and a -1.05 to $-1.52-$ fold DR for the 5 genes UGT73C6, 3MaT, CYP71B34, CYP71B35 and CYP81Q32 (Table 3). Four genes belonged to transcription factors (TFs) with a 3.76-, 1.15-, 1.19- and 2.40-fold UR for RL1, RL6, MYB90 and MYB114, respectively (Table 4).

Table 3. DEGs involved in flavonoid biosynthesis and their RPKM values in M1 vs. M2.

\begin{tabular}{|c|c|c|c|}
\hline Gene Name & Protein Name & SwissProt ID & $\log _{2} \mathrm{FC}(\mathrm{M} 1$ vs. M2) \\
\hline CHS1 & Chalcone synthase 1 & Q9ZS41 & 8.63 \\
\hline $\mathrm{CHI3}$ & $\begin{array}{l}\text { Probable chalcone-flavonone } \\
\text { isomerase } 3\end{array}$ & Q8VZW3 & 1.06 \\
\hline $\mathrm{F} 3 \mathrm{H}$ & Flavanone 3-dioxygenase & Q7XZQ7 & 1.97 \\
\hline DFR & Dihydroflavonol 4-reductase & P51105 & 6.50 \\
\hline ANS & $\begin{array}{l}\text { Leucoanthocyanidin } \\
\text { dioxygenase }\end{array}$ & P51091 & 7.51 \\
\hline CGT & UDP-glycosyltransferase 13 & A0A0M4KE44 & 1.06 \\
\hline GT6 & $\begin{array}{l}\text { UDP-glucose flavonoid } \\
\text { 3-O-glucosyltransferase } 6\end{array}$ & Q2V6K0 & 2.25 \\
\hline UGT85A8 & UDP-glycosyltransferase $85 \mathrm{~A} 8$ & Q6VAB3 & 1.31 \\
\hline UGT73C6 & UDP-glycosyltransferase 73C6 & Q9ZQ95 & -1.52 \\
\hline F3GT1 & $\begin{array}{l}\text { Anthocyanidin } \\
\text { 3-O-galactosyltransferase F3GT1 } \\
\text { Malonyl-coenzyme }\end{array}$ & A0A2R6Q8R5 & 1.17 \\
\hline $3 \mathrm{MaT}$ & $\begin{array}{l}\text { A:anthocyanin 3-O-glucoside- } 6^{\prime \prime} \text { - } \\
\text { O-malonyltransferase } \\
\text { Pelargonidin }\end{array}$ & Q8GSN8 & -1.28 \\
\hline P5MaT & $\begin{array}{l}\text { 3-O-(6-caffeoylglucoside) } \\
\text { 5-O-(6-O-malonylglucoside) } \\
4^{\prime \prime \prime} \text {-malonyltransferase }\end{array}$ & Q6TXD2 & 1.21 \\
\hline CYP71A1 & Cytochrome P450 71A1 & P24465 & 1.19 \\
\hline CYP71A9 & Cytochrome P450 71A9 & O81970 & 1.04 \\
\hline CYP71D313 & Cytochrome P450 CYP71D313 & $\mathrm{H} 2 \mathrm{DH} 20$ & 2.21 \\
\hline CYP71B26 & Cytochrome P450 71B26 & Q9LTL0 & 1.77 \\
\hline CYP71B36 & Cytochrome P450 71B36 & Q9LIP4 & 1.33 \\
\hline CYP72A219 & Cytochrome P450 CYP72A219 & $\mathrm{H} 2 \mathrm{DH} 21$ & 1.21 \\
\hline CYP736A12 & Cytochrome P450 CYP736A12 & H2DH18 & 1.37 \\
\hline CYP76AD1 & Cytochrome P450 76AD1 & I3PFJ5 & 2.59 \\
\hline CYP76A2 & Cytochrome P450 76A2 & P37122 & 1.15 \\
\hline СУР77АЗ & Cytochrome P450 77A3 & O48928 & 1.79 \\
\hline CYP71B34 & Cytochrome P450 71B34 & Q9LIP6 & -1.05 \\
\hline CYP71B35 & Cytochrome P450 71B35 & Q9LIP5 & -1.35 \\
\hline CYP81Q32 & Cytochrome P450 81Q32 & W8JMU7 & -1.12 \\
\hline
\end{tabular}

Table 4. Differentially expressed transcription factor (TF) involved in flavonoid biosynthesis and their RPKM values in M1 vs. M2.

\begin{tabular}{llll}
\hline Gene Name & Protein Name & SwissProt ID & $\log _{2}$ FC(M1 vs. M2) \\
\hline RL1 & Protein RADIALIS-like 1 & F4JVB8 & 3.76 \\
RL6 & Protein RADIALIS-like 6 & Q1A173 & 1.15 \\
MYB90 & Transcription factor MYB90 & Q9ZTC3 & 1.19 \\
MYB114 & Transcription factor MYB114 & Q9FNV8 & 2.40 \\
\hline
\end{tabular}

\subsection{Network of DAFs Regulated by DEGs}

The 26 DAFs and 25 DEGs (exclude 4 TFs) were connected based on the flavonoid biosynthetic pathway analyzed by $\mathrm{KO}$ enrichment and biological function of proteins on the SwissProt database, and the proposed biosynthetic pathway is shown in Figure 7. 
Flavonoids are synthesized via the phenylpropanoid pathway. Briefly, the upstream metabolite 4-coumaroyl-CoA is formed from phenylalanine by the catalyzation of PAL, C4H and 4CL. The 4-coumaroyl-CoA is converted into two metabolites, isoliquiritigenin and naringenin chalcone, by the catalyzation of $\mathrm{CHS}$, then respectively transformed to liquiritigenin and naringenin by the catalyzation of $\mathrm{CHI}$. In the sub-pathway of isoflavonoid biosynthesis, 13 cytochrome P450 monooxygenases (CYPs) are involved, and butin-7$\mathrm{O}$-glucoside (21) is produced by the catalyzation of $\mathrm{F}^{\prime} \mathrm{H}$. In the sub-pathway of anthocyanin biosynthesis, 10 genes (F3H, DFR, ANS, CGT, GT6, UGT85A8, UGT73C6, F3GT1, $3 \mathrm{MaT}$ and $\mathrm{P5MaT}$ ) and four anthocyanins are involved, and pelargonidin-3-O-glucoside5-O-arabinoside (23), cyanidin-3-O-glucoside (24), cyanidin-3-O-sambubioside (25) and peonidin-3-O-sambubioside (26) are formed. Under the catalyzation of FLS and F3' $\mathrm{H}$, the metabolites kaempferol and quercetin are produced, then 2 kaempferol-derivatives (18 and 19) and 14 quercetin-derivatives (1 to 14) are formed. In addition, the chrysoeriol-5-Oglucoside (15), naringenin-7-O-glucoside (16), hesperetin-5-O-glucoside (17) and isosalipurposide (20) are also mapped in the phenylpropanoid pathway.

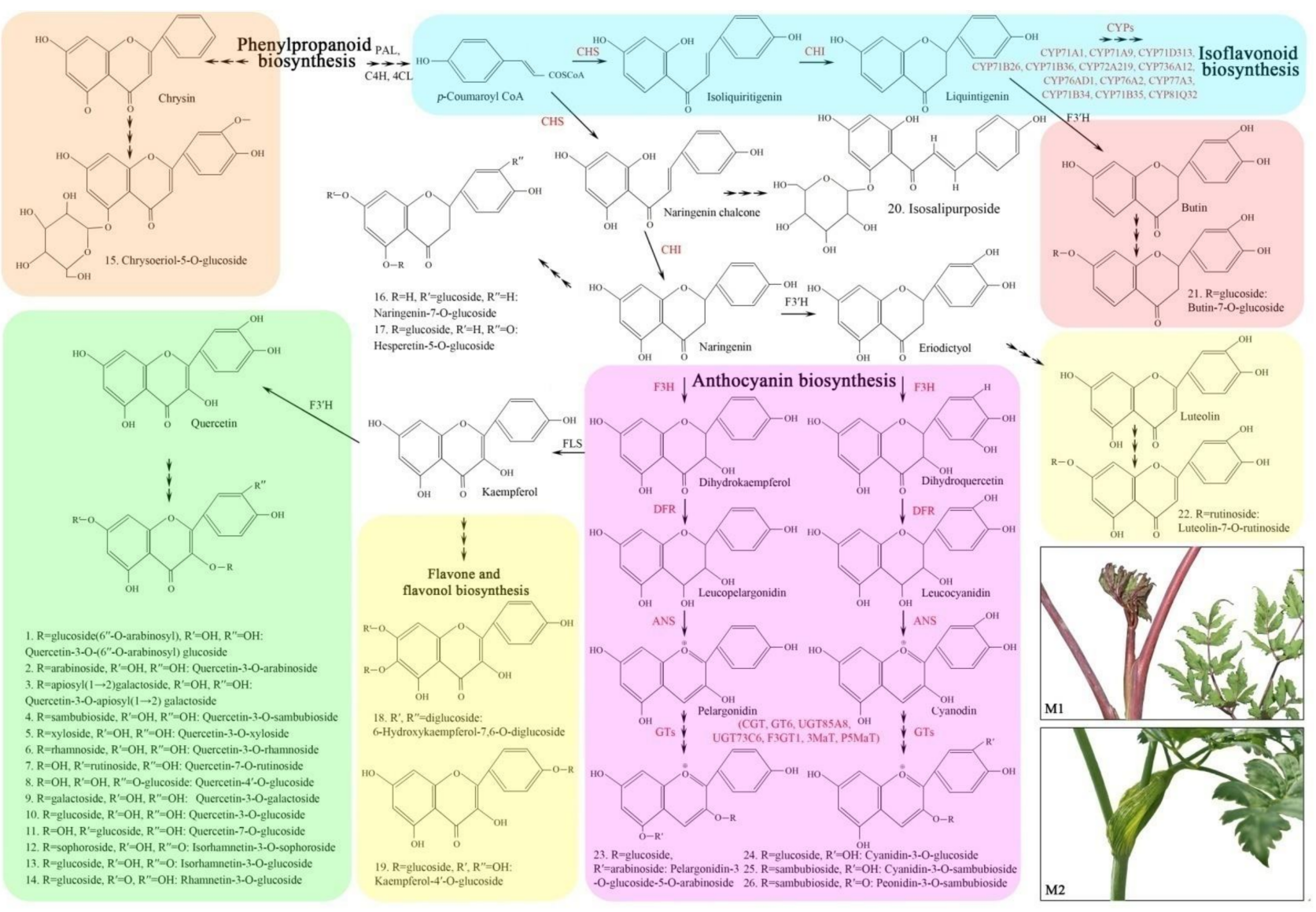

Figure 7. Proposed network of the DAFs regulated by the DEGs in M1 and M2. The 26 DAFs are listed from No.1 to No.26 (Table 1), and the enzymes encoded by the 25 DEGs are colored in red.

\section{7. qRT-PCR Validation of Candidate Genes Involved in Flavonoid Biosynthesis}

As shown in Figure 7, 25 DEGs were mapped in the pathway of flavonoid biosynthesis, with 20 UR and 5 DR in M1 vs. M2 (Table 3). Among them, 22 genes (20 UR and 2 DR) were selected to qRT-PCR validation, and their relative expression levels (RELs) were consistent with RPKM values (Table 3, Figure 8). 

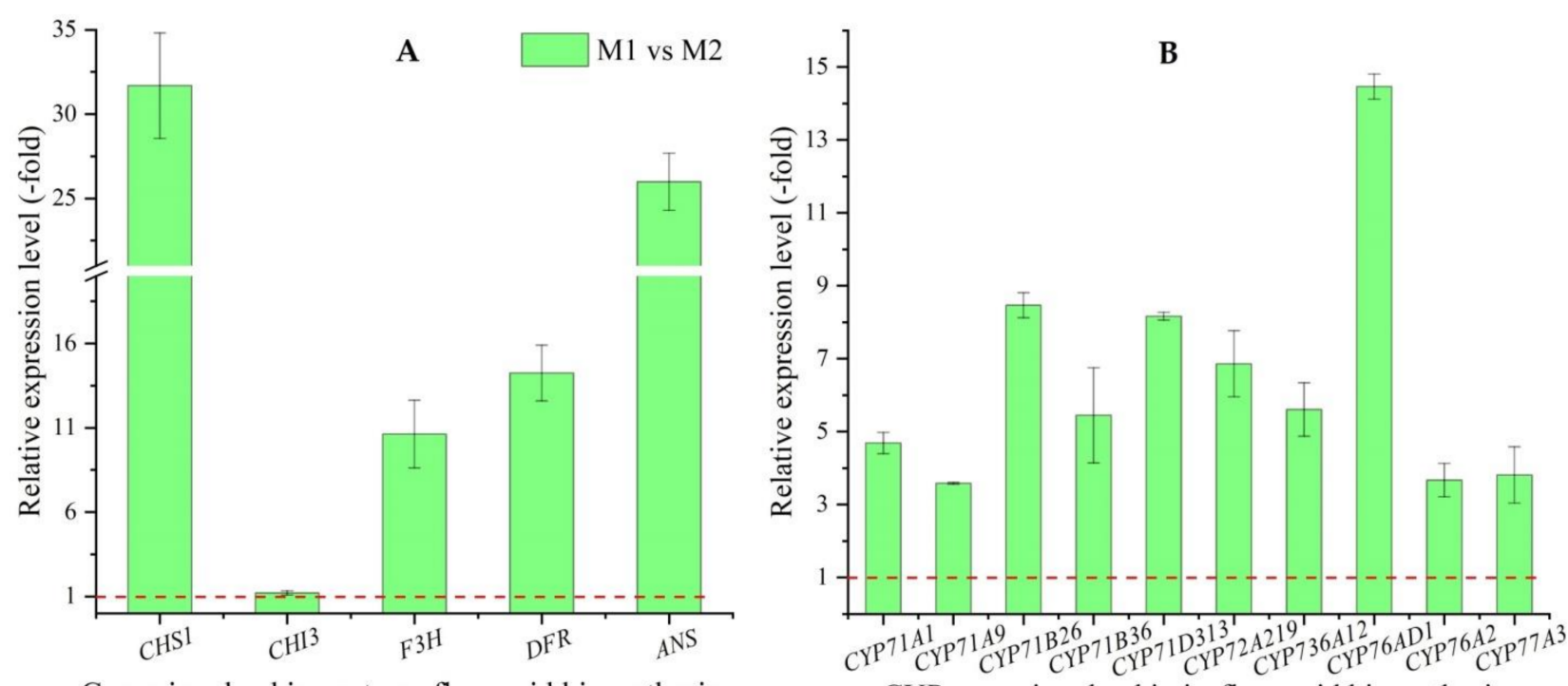

Genes involved in upsteam flavonoid biosynthesis

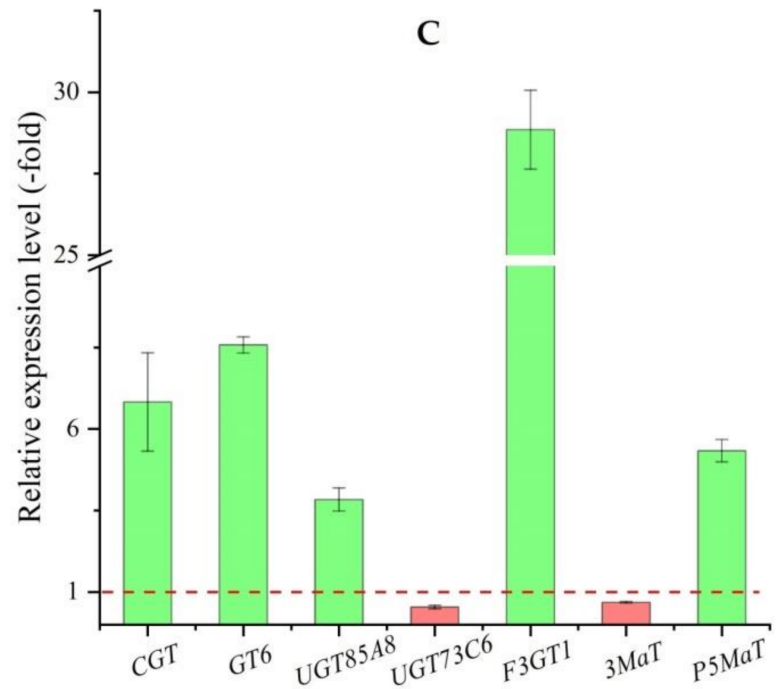

Genes involved in anthocyanin biosynthesis

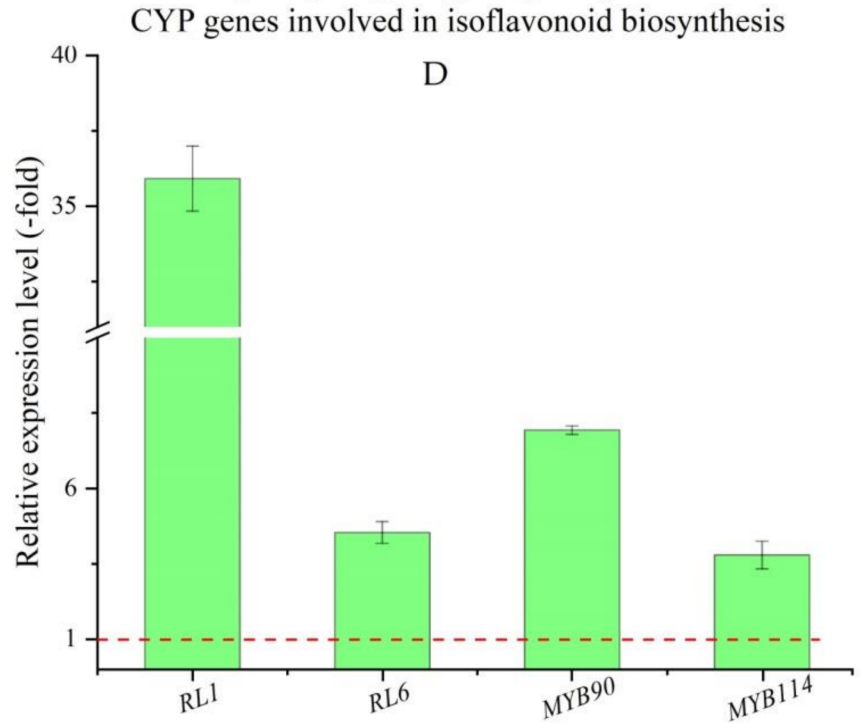

TFs involved in anthocyanin biosynthesis

Figure 8. The RELs of genes involved in flavonoid biosynthesis (A), isoflavonoid biosynthesis (B), anthocyanin biosynthesis (C), and TFs (D) in M1 vs. M2, as determined by qRT-PCR (mean \pm SD, $n=3$ ). The column highlighted in green represents genes favoring flavonoid biosynthesis and red represents genes disfavoring flow.

Specifically, five genes directly participating in upstream flavonoid biosynthesis included CHS1, CHI3, F3H, DFR and ANS, their RELs exhibited a 31.70-, 1.21-, 10.63-, 14.24and 26.00-fold UR, respectively, in M1 vs. M2 (Figure 8A).

Thirteen CYP genes participate in isoflavonoid biosynthesis with 10 UR genes including CYP71A1, CYP71A9, CYP71D313, CYP71B26, CYP71B36, CYP72A219, CYP736A12, CYP76AD1, CYP76A2 and CYP77A3, as well as 3 DR genes including CYP71B34, CYP71B35 and CYP81Q32 (Figure 7), The RELs of the 10 UR genes exhibited a 4.09-, 3.58-, 8.47-, 5.45-, 8.16-, 6.86-, 5.61-, 14.47-, 3.67- and 3.81-fold, respectively, in M1 vs. M2 (Figure 8B).

Seven genes directly participating in anthocyanin biosynthesis included CGT, GT6, UGT85A8, UGT73C6, F3GT1, 3MaT and P5MaT. The RELs of the five genes CGT, GT6, UGT85A8, F3GT1 and P5MaT exhibited a 6.83-, 8.58-, 3.84-, 28.86- and 5.33-fold UR, while the two genes UGT73C6 and 3MaT exhibited a 0.54- and 0.68-fold DR, respectively, in M1 vs. M2 (Figure 8C). 
Four TFs participating in regulating anthocyanin biosynthesis included $R L 1, R L 6$, MYB90 and MYB114, their RELs exhibited a 35.92-, 4.54-, 7.90-, and 3.75-fold UR, respectively, in M1 vs. M2 (Figure 8D).

\section{Discussion}

Accumulation of secondary metabolites is not only influenced by environmental factors (e.g., temperature, light, the supply of water and minerals) but also genotypes (e.g., variety, strain and cultivar) [21-23]. Previous studies have demonstrated that there is a significant difference of secondary metabolites among the three Angelica species: A. sinensis (Oliver) Diels, A. dahurica (Fisch. ex Hoffn) Benth, et Hook. F, and A. pubescens Maxim [5]. A higher root yield of M1 than M2 was observed [16-18]. In this study, the flavonoid and anthocyanin contents were greater in M1 than M2, 26 DAFs (19 UR and 7 DR) and 29 DEGs (24 UR and 5 DR) involved in flavonoid biosynthesis were observed in M1 vs. M2.

Flavonoids are widely distributed secondary metabolites with different metabolic functions in plants, such as providing colors attractive to plant pollinators, promoting physiological survival, and protecting plants from fungal pathogens and UV-B radiation; meanwhile, flavonoids possess antifungal, antioxidant and anticancer activities [24,25]. In this study, a 1.48 increase of flavonoid content was observed in M1 compared to M2 (Figure 1), suggesting that the adaptation ability of M1 to environmental conditions is stronger than that of M2, which is consistent with previous investigations that the yield and tolerance of M1 is greater and stronger than that of M2 in the field [16-18]. Additionally, a 2.57-fold greater anthocyanin content was observed in M1 compared to M2 (Figure 1), which can describe the difference of stem-color formation for M1 with purple stem and M2 with green stem. Several studies have reported that the contents of flavonoids and anthocyanins play a positive role in pigmentation $[26,27]$.

Currently, more than 6000 different flavonoids have been identified from plants, and they can be classified into six major subgroups, including chalcones, flavones, flavonols, flavandiols, anthocyanins, and proanthocyanidins or condensed tannins [28]. In this study, 131 flavonoids were identified from M1 and M2 by LC-ESI-MS/MS analysis including flavonols (51), flavonoid (36), flavanols (8), chalcones (7), dihydroflavone (7), dihydroflavonol (7), isoflavones (6), anthocyanins (5), and flavonoid carbonoside (4); and 26 of them were differentially accumulated in M1 vs. M2 (Figure 2 and Table 1). The 26 DAFs were enriched in five pathways and mapped on the phenylpropanoid pathway (Figure 3, Figure 7).

Extensive experiments have demonstrated that the expression of genes encoding enzymes and TFs is responsible for the formation of flavonoid structures and their subsequent modification reactions [29]. In this study, 29 genes participating in flavonoid biosynthesis were screened from the 2210 DEGs in M1 vs. M2; the specific role of the 29 genes has been linked with the 26 DAFs and mapped on the phenylpropanoid pathway (Figures 5 and 7; Tables 3 and 5).

Table 5. Primer sequence of candidate genes used for qRT-PCR validation.

\begin{tabular}{|c|c|c|c|}
\hline Gene Name & Primer Sequences $\left(5^{\prime}\right.$ to $\left.3^{\prime}\right)$ & & Amplicon Size (bp) \\
\hline \multirow{2}{*}{$A C T$} & Forward: TGGTATTGTGCTGGATTCTGGT & \multirow{2}{*}{109} & \\
\hline & Reverse: TGAGATCACCACCAGCAAGG & & \\
\hline \multicolumn{4}{|c|}{ Flavonoid biosynthesis (22) } \\
\hline \multirow{2}{*}{ CHS1 } & Forward: CATTTCGGGGGCCTAACGAT & \multirow{2}{*}{197} & \\
\hline & Reverse: CCCAACCTCCCGAAGATGAC & & \\
\hline \multirow{2}{*}{$\mathrm{CHI3}$} & Forward: CACGGACATTGAGATACACTTCC & \multirow{2}{*}{111} & \\
\hline & Reverse: TCTCCAGTTTTTCCCTTCCAGT & & \\
\hline
\end{tabular}


Table 5. Cont.

\begin{tabular}{|c|c|c|c|}
\hline Gene Name & Primer Sequences $\left(5^{\prime}\right.$ to $\left.3^{\prime}\right)$ & & Amplicon Size (bp) \\
\hline \multirow{2}{*}{$\mathrm{F} 3 \mathrm{H}$} & Forward: AGTGAGAAGTTGATGGCGCT & \multirow{2}{*}{160} & \\
\hline & Reverse: GTCCCAGTGTCAAGTCAGGT & & \\
\hline \multirow{2}{*}{$D F R$} & Forward: ACAGCACTATCACCGCTCAC & \multirow{2}{*}{134} & \\
\hline & Reverse: ATGTATCTTCCCTGCGCTGT & & \\
\hline \multirow{2}{*}{ ANS } & Forward: GGCCTCAAGTGCCTACAGTT & \multirow{2}{*}{169} & \\
\hline & Reverse: TGTCCAGCCACTCTAACACG & & \\
\hline \multirow{2}{*}{ CGT } & Forward: GCAGCCCGCAAAATCTGTAG & \multirow{2}{*}{163} & \\
\hline & Reverse: ACGCAACCCTTCCTTGTCTT & & \\
\hline \multirow{2}{*}{ GT6 } & Forward: GTGCCACAGGTGACGATTCT & \multirow{2}{*}{173} & \\
\hline & Reverse: ACTCCCAGTCCCAАСТССТT & & \\
\hline \multirow{2}{*}{ UGT85A8 } & Forward: ATGCAGTATCGCCAACTCGT & \multirow{2}{*}{111} & \\
\hline & Reverse: GTCTTTCATTCCAGGAGCCCA & & \\
\hline \multirow{2}{*}{ UGT73C6 } & Forward: GTATGGGCAGTAAGGGCTGG & \multirow{2}{*}{110} & \\
\hline & Reverse: GCCCAACCACGGATCAAAAG & & \\
\hline \multirow{2}{*}{ F3GT1 } & Forward: GCTTTGGAACTGTGGCGATG & \multirow{2}{*}{165} & \\
\hline & Reverse: AGGCCACGATTTTTCCGGTT & & \\
\hline \multirow{2}{*}{$3 M a T$} & Forward: CTCCGTGACATCTCTGCCTC & \multirow{2}{*}{175} & \\
\hline & Reverse: AGCCAACGGAGTGAAGTGTT & & \\
\hline \multirow{2}{*}{ P5MaT } & Forward: AGGCGAAAAAGGGGTGGAAT & \multirow{2}{*}{193} & \\
\hline & Reverse: GCACCAGTCGGTAAACAAGC & & \\
\hline \multirow{2}{*}{ CYP71A1 } & Forward: GTTTACGTGAGTGCATGGGC & \multirow{2}{*}{138} & \\
\hline & Reverse: TGCCCCAAAAGGAACCAACT & & \\
\hline \multirow{2}{*}{ CYP71A9 } & Forward: CAATGCTTGGGCAACAAACG & \multirow{2}{*}{153} & \\
\hline & Reverse: TTTCTGCTTCTCGGATAGGGC & & \\
\hline \multirow{2}{*}{ CYP71D313 } & Forward: GCTTGGTGAGATCCCTCTGG & \multirow{2}{*}{108} & \\
\hline & Reverse: TCACCAAGTACAAGTCCTGGC & & \\
\hline \multirow{2}{*}{ CYP71B26 } & Forward: TGTTGTGTGGGCCATGACTT & 157 & \\
\hline & Reverse: TCTCATTGCСТССТТСАССАС & & \\
\hline CҮP71B36 & Forward: GGGCTGAGAACAGGTCAAGT & 199 & \\
\hline & Reverse: CTTGTATCGGCTCCTGCAAC & & \\
\hline Cציברים & Forward: TTGCTCGTGTGGACTGTTGT & 186 & \\
\hline & Reverse: TCGTAGAAGCATACCTGCCG & & \\
\hline CYP736A12 & Forward: GGAAACCTCCCTCATCGCTC & 167 & \\
\hline & Reverse: GCCTCAAATTCTGGACGGCT & & \\
\hline SYP76AD1 & Forward: AATCGGAGCGAAAGGAAGCC & 132 & \\
\hline & Reverse: ACGTTGGTCACCGTTTTGTG & & \\
\hline CYP76A2 & Forward: GCAGGTTTCACCGAGAGTGT & 164 & \\
\hline C11 & Reverse: TGTTGCCTCTCCATCACACG & & \\
\hline$C Y P 7743$ & Forward: TTAGCAGTGCGGATTTGGCT & & \\
\hline & Reverse: CGGACCGTAGAGTGAGGAGT & & \\
\hline
\end{tabular}


Table 5. Cont.

\begin{tabular}{|c|c|c|c|}
\hline Gene Name & Primer Sequences $\left(5^{\prime}\right.$ to $\left.3^{\prime}\right)$ & & Amplicon Size (bp) \\
\hline \multicolumn{4}{|c|}{ MYB transcription factor (4) } \\
\hline \multirow{2}{*}{$R L 1$} & Forward: TTGAAAAGGCTCTGGCTGTGT & \multirow{2}{*}{127} & \\
\hline & Reverse: CTGATGTCTGCCACGAGGATT & & \\
\hline \multirow{2}{*}{$R L 6$} & Forward: GCGTAACTGTGGCTCTACCT & \multirow{2}{*}{102} & \\
\hline & Reverse: GCTATGTTATGCCAGCGGTC & & \\
\hline \multirow{2}{*}{ MYB114 } & Forward: TTCGTAAGGGTGCATGGTGT & \multirow{2}{*}{140} & \\
\hline & Reverse: AAGCCACCTCAGTCTACAGC & & \\
\hline \multirow{2}{*}{ MYB90 } & Forward: AAAGGCACAAGCCTACCCTG & \multirow{2}{*}{136} & \\
\hline & Reverse: CTGGGGGCAGTGTCTTCATC & & \\
\hline
\end{tabular}

Flavonoids are synthesized via the phenylpropanoid pathway with transforming phenylalanine into 4-coumaroyl-CoA, and then entering the sub-pathways of flavonoid biosynthesis under the coordinated regulation of key genes (Figure 7). In this study, five genes that directly participate in upstream flavonoid biosynthesis include CHS1, CHI3, F3H, DFR and ANS, which is responsible for sequentially converting 4-coumaroyl$\mathrm{CoA}$ to naringenin chalcone, naringenin, dihydrokaempferol, leucoanthocyanidin and pelargonidin [30-32].

CYPs play diverse roles in metabolism including the synthesis of secondary metabolites (e.g., flavonoids, alkaloids and lignan) [33,34]. Previous studies have found that the overexpression of $C Y P_{s}$ genes promotes flavonoid and pigment biosynthesis [35,36]. In this study, 13 CYPs genes directly participate in isoflavonoid biosynthesis with 10 UR (Figure 7; Table 3), which will enhance the flavonoid biosynthesis and greater accumulation in M1 compared to M2 (Figure 1).

UDP-glycosyltransferases (UGTs) is one of the glycosyltransferases that comprise a highly divergent and polyphyletic multigene family involved in widespread glycosylation of plant secondary metabolites (e.g., anthocyanins) [37]. In this study, five UGTs genes were observed to participate in anthocyanin biosynthesis. Previous studies have found that CGT is involved in the biosynthesis of mangiferin [38], GT6 is involved in the formation of flavonol 3-O-glucosides [39], UGT85A8 is involved in glycosylate diterpenes or flavonols, UGT73C6 is involved in flavonol biosynthetic process while possessing low quercetin 3-Oglucosyltransferase, 7-O-glucosyltransferase and 4'-O-glucosyltransferase activities [40,41], and F3GT1 is involved in anthocyanin biosynthesis by catalyzing the galactosylation of cyanidin [42]. Meanwhile, two genes encoding malonyltransferase (MaT) that is also involved in anthocyanin biosynthesis include: $3 M a T$ involved in the transfer of the malonyl group from malonyl-CoA to pelargonidin 3-O-glucoside to produce pelargonidin 3-O-6"$\mathrm{O}$-malonylglucoside [43], and P5MaT involved in the transfer of the malonyl group from malonyl-CoA to the $4^{\prime \prime \prime}$-hydroxyl group of the 5-glucosyl moiety of anthocyanins [44].

TFs play a great role in controlling cellular processes and MYB TF family is involved in controlling various processes such as responses to biotic and abiotic stresses, development, and metabolism, etc [45]. Several investigations have found that the overexpression of MYB TFs promote flavonoid and anthocyanin biosynthesis [46,47]. In this study, 4 MYB TFs were observed to be in involved in anthocyanin biosynthesis. The two TFs RL1 and $R L 6$ as a member of the MYB-related gene family may regulate the anthocyanin biosynthesis [48]. The two TFs MYB90 and MYB114 are transcription activators, when associated with BHLHs/MYCs, EGL3, or GL3, they promote the synthesis of phenylpropanoid-derived compounds such as anthocyanins $[49,50]$. 


\section{Materials and Methods}

\subsection{Plant Material}

Functional leaves and petioles of two-year-old Angelica sinensis [two cultivars: Mingui 1 (M1) with purple stem and Mingui 2 (M2) with green stem, Figure S10] were collected from the city-owned breeding garden located in Shangconggou village, Huichuan Town, Weiyuan County, Dingxi City (2507 m a.s.1.; $\left.35^{\circ} 2^{\prime} 39^{\prime \prime} \mathrm{N}, 104^{\circ} 1^{\prime} 55^{\prime \prime} \mathrm{E}\right)$ of Gansu province, China in July 2020. The two cultivars were identified by Professor Ling Jin (Gansu University of Chinese Medicine, Lanzhou, China). Voucher specimens (M1: 20190725GSWYMG1, M2: 20190725GSWYMG2) were deposited in the herbarium of College of Pharmacy, Gansu University of Chinese Medicine, Lanzhou, China. During the growth stages, the two cultivars were maintained with the same field management conditions. The collected samples (leaves and petioles $=1: 1, \mathrm{~g} / \mathrm{g}$ fresh weight; $\mathrm{n}=20$ plants) were immediately frozen in liquid nitrogen for total flavonoid and anthocyanin measurement, metabolomic and transcriptomic analysis.

\subsection{Chemicals}

Standards of metabolites used for UPLC analysis were purchased from BioBioPha (Kunming, Yunnan, China) and Sigma-Aldrich (St Louis, MO, CA, USA). All chemicals and reagents (e.g., $\mathrm{AlCl}_{3}$, catechin, ethanol, $\mathrm{HCL}$, methanol, $\mathrm{NaNO}_{2}$ and $\mathrm{NaOH}$ ) were of analytical grade and purchased from Merck, Germany. Trizol reagent, RT Kit and SuperReal PreMix were purchased from Tiangen, China.

\subsection{Measurement of Total Flavonoid and Anthocyanin Contents}

\subsubsection{Measurement of Total Flavonoid Content}

Fresh samples (0.5 g) were placed in ethanol $(5 \mathrm{~mL}, 95 \% \mathrm{v} / \mathrm{v})$ and ground, the homogenate was centrifuged at $5000 \mathrm{r} / \mathrm{min}$ for $10 \mathrm{~min}$ at $4{ }^{\circ} \mathrm{C}$ and re-extracted twice more. The extracts were increased to $20 \mathrm{~mL}$ with ethanol $(95 \% \mathrm{v} / \mathrm{v})$. Total flavonoids content was measured according to a $\mathrm{NaNO}_{2}-\mathrm{AlCl}_{3}-\mathrm{NaOH}$ method [51,52]. Briefly, extracts $(150 \mu \mathrm{L})$ were added in $\mathrm{ddH}_{2} \mathrm{O}(2 \mathrm{~mL})$ and $\mathrm{NaNO}_{2}(5 \% \mathrm{w} / \mathrm{v}, 0.3 \mathrm{~mL})$. After the mixture agitating for $5 \mathrm{~min}, \mathrm{AlCl}_{3}(10 \% \mathrm{w} / \mathrm{v}, 0.3 \mathrm{~mL})$ was added and reacted for $1 \mathrm{~min}$, then $\mathrm{NaOH}(1.0$ $\mathrm{mol} / \mathrm{L}, 2 \mathrm{~mL}$ ) was added to stop the reaction. Absorbance readings were taken at $510 \mathrm{~nm}$ using a spectrometer. Total flavonoid content was calculated based on a standard curve and expressed as mg of catechin.

\subsubsection{Measurement of Anthocyanin Content}

Anthocyanins content was measured according to a previous protocol [53]. Fresh samples $(0.5 \mathrm{~g})$ were placed in methanol $(5 \mathrm{~mL}, 0.1 \% \mathrm{HCL} v / v)$ and ground, the homogenate was centrifuged at $5000 \mathrm{r} / \mathrm{min}$ for $30 \mathrm{~s}$ at $4{ }^{\circ} \mathrm{C}$ and re-extracted twice more. The extracts

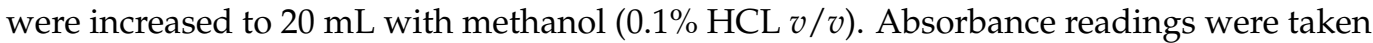
at $530 \mathrm{~nm}$ using a spectrometer. Anthocyanins content was evaluated based on a relative expression level compared to the blank control.

\subsection{Metabolomic Analysis}

\subsubsection{Sample Preparation and Extraction}

The freeze-dried samples were ground in a mixer mill with zirconia beads for $1.5 \mathrm{~min}$ at $30 \mathrm{~Hz}$. The powder $(0.1 \mathrm{~g})$ was added into methanol $(70 \% v / v, 1 \mathrm{~mL})$ and extracted for $12 \mathrm{~h}$ at $4{ }^{\circ} \mathrm{C}$, and then the homogenate was centrifuged at $10,000 \mathrm{r} / \mathrm{min}$ for $10 \mathrm{~min}$ at $4{ }^{\circ} \mathrm{C}$. The supernatant was filtrated with a $0.22 \mu \mathrm{m}$ durapore membrane for LC-MS/MS analysis.

\subsubsection{UPLC Analysis}

The metabolites were firstly analyzed using a LC-ESI-MS/MS system (UPLC, Shimpack UFLC CBM-20A, Shimadzu, Japan). Extracts $(5 \mu \mathrm{L})$ were analyzed using a Waters ACQUITY UPLC HSS T3 C18 column $(100 \mathrm{~mm} \times 2.1 \mathrm{~mm}, 1.8 \mu \mathrm{m}$; m; column temperature $\left.40^{\circ} \mathrm{C}\right)$. Acetic acid $(0.04 \% v / v, \mathrm{~A})$-acetonitrile (B) made up the mobile phase with 
gradient elution: 5\% B (0-11 $\mathrm{min}), 95 \% \mathrm{~B}(11-12 \mathrm{~min})$ and 5\% B (12.1-15 $\mathrm{min})$ at a flow rate of $0.4 \mathrm{~mL} / \mathrm{min}$. Quality control samples were mixed by all the samples to detect reproducibility of the whole experiments (Figures S11 and S12).

\subsubsection{MS/MS Analysis}

The effluent from UPLC was analyzed using an AB SCIEX QTRAP 4500 and Triple Quad 4500 Systems (AB SCIEX, Boston, MA, USA) equipped with an ESI-Turbo Ion-Spray interface and operated in a positive ion mode. The operation parameters were as follows: ESI source temperature $550{ }^{\circ} \mathrm{C}$, ion spray voltage $5500 \mathrm{~V}$, curtain gas 25 psi, collisionactivated dissociation set 5 pis. Triple quadrupole scans were acquired as MRM experiments with optimized de-clustering potential and collision energy CE for each individual multiple reaction monitoring (MRM) transitions. The $m / z$ range was set between 50 and 1000 .

\subsubsection{Metabolites Identification}

Metabolites were identified using internal and public databases (MassBank, KNApSAcK, HMDB, MoTo DB and METLIN) and comparing $m / z$ values, retention time, and the fragmentation patterns with the standards.

\subsubsection{Differential Metabolites Analysis}

The accumulation level of metabolites was ranked using a variable importance in projection (VIP) scores of orthogonal projection to latent structures-discriminant analysis (OPLS-DA). The level of differential accumulation between M1 and M2 was determined with a criterion of VIP $\geq 1$ and $t$-test $p \leq 0.05$.

\subsection{Isoform Sequencing and Transcriptomic Analysis}

\subsection{1. cDNA Library Construction and Single Molecular Real-Time (SMRT) Sequencing}

Total RNA was extracted using a Trizol reagent according to the manufacturer's protocol. The quality of extracted RNA was determined using a Agilent 2100 Bioanalyzer and agarose gel electrophoresis. mRNA was enriched by Oligo (dT) magnetic beads and transcribed into cDNA using a Clontech SMARTer cDNA Synthesis Kit. Then the cDNA was amplified by PCR for 13 cycles to prepare for the next SMRTbell library construction. The $>5 \mathrm{~kb}$ size sequence was ligated to the sequencing adapters. The SMRTbell template was applied and sequenced on a PacBio SequelII platform (Gene Denovo Biotechnology Co., Ltd., Guangzhou, China).

\subsubsection{Isoform Data Processing}

The raw sequencing reads of cDNA libraries were analyzed using a isoform sequencing (Iso-Seq) system [54]. Briefly, high quality CCS were extracted and then the FLNC reads were obtained after removing the primers, barcodes, poly (A) tail trimmings and concatemers. The FLNC reads were clustered to generate the entire isoform, which was used for sequences correction. Finally, isoforms were BLAST analyzed against the Nr database, isoforms were annotated against the databases including: KEGG, KOG and Swiss-Prot.

\subsubsection{Transcriptomic Analysis and DEGs Identification}

Total RNA was extracted using a Trizol reagent according to the manufacturer's protocol. The processes of enrichment by Oligo (dT) magnetic beads, fragmentation by ultrasonic, reverse transcription by a cDNA Synthesis Kit, synthesis of the second-strand cDNA by PCR amplification as well as purification of cDNA fragments by end-repairing and adapter-connecting were conducted according to previous protocols [55]. RNA-seq was performed by an Illumina HiSeqTM 4000 platform (Gene Denovo Biotechnology Co., Ltd., Guangzhou, China). Raw reads were filtered according to previous protocols (Li et al., 2008). Clean reads was assembled using Trinity [56]. The expression level of each transcript was normalized to RPKM values [57], and the differential expression level between M1 and 
M2 was determined with a criteria of $\mid \log _{2}$ (fold-change) $\mid \geq 1$ and $p \leq 0.05$ by DESeq2 software and the edgeR package [58,59].

\section{6. qRT-PCR Validation of Genes Involved in Flavonoid Biosynthesis}

The primer sequence (Table 5) was designed via a primer-blast in NCBI and synthesized by reverse transcription (Sangon Biotech Co., Ltd., Shanghai, China). First cDNA was synthesized using a RT Kit. PCR amplification was performed using a SuperReal PreMix. Melting curve was analyzed at $72{ }^{\circ} \mathrm{C}$ for $34 \mathrm{~s}$. Actin gene was used as a reference control. The RELs of gene were calculated using a $2^{-\Delta \Delta C t}$ method [60].

\subsection{Statistical Analysis}

All experiments were performed in three biological replicates in this study. A $t$-test in SPSS 22.0 was performed for independent treatments with $p<0.05$ as the basis for statistical differences.

\section{Conclusions}

From the above observations, the flavonoid and anthocyanin contents in the cultivar M1 of A. sinensis were greater than in M2, which rely on the up-regulation of genes involved in flavonoid and anthocyanin biosynthesis. The difference of stem color formation between M1 and M2 results from the anthocyanin differential accumulation as well as the genes' differential expression.

Supplementary Materials: The following supporting information can be downloaded online. Figure S1: PCA of M1 (PSAS) and M2 (GSAS) as well as quality control (QC) samples; Figure S2: OPLS-DA of M1 and M2; Figure S3: Cluster heat map of the 26 DAFs in M1 vs. M2; Figure S4: Length distribution of high-fidelity reads (A), high-quality isoforms (B) and full-length isoforms (C); Figure S5: RPKM distribution of M1 and M2; Figure S6: Violin plot of expression in M1 and M2; Figure S7: PCA analysis of M1 and M2; Figure S8: Pearson Heat-map correlation between M1 and M2; Figure S9: GO classification of the DAGs in M1 vs. M2; Figure S10: Aerial-parts characteristics of the two Angelica sinensis cultivars: M1 with purple stem and M2 with green stem; Figure S11: Representative total-ion-chromatogram (TIC) of QC sample; Figure S12: Representative TIC of MRM metabolites detection of QC sample.

Author Contributions: T.Z.: Formal analysis, investigation and writing —original draft preparation; M.Z.: Formal analysis and validation; H.S.: Data curation and validation; M.L. (Meiling Li): Formal analysis; Y.W.: Formal analysis; L.J.: Project administration, resources and supervision; M.L. (Mengfei Li): Conceptualization and writing-review and editing. All authors have read and agreed to the published version of the manuscript.

Funding: This research was funded by National Natural Science Foundation of China (81360615 and 32160083), Innovation Base and Talent Plan of Gansu Province of China (20JR5RA182), Double FirstClass initiative projects of Gansu Province of China (GSSYLXM-05), Gansu University of Chinese Medicine (2021KCZD-4), Assurance Project of Ecological Planting and Quality of Daodi Herbs (202103003), and State Key Laboratory of Aridland Crop Science Gansu Agricultural University (GSCS-2021-Z03).

Informed Consent Statement: Not applicable.

Data Availability Statement: The datasets are publicly available at NCBI with Sequence Read Archive (SRA) accession: SRR16993328 to SRR16993332.

Conflicts of Interest: All the authors declare that they have no conflicts of interest. 


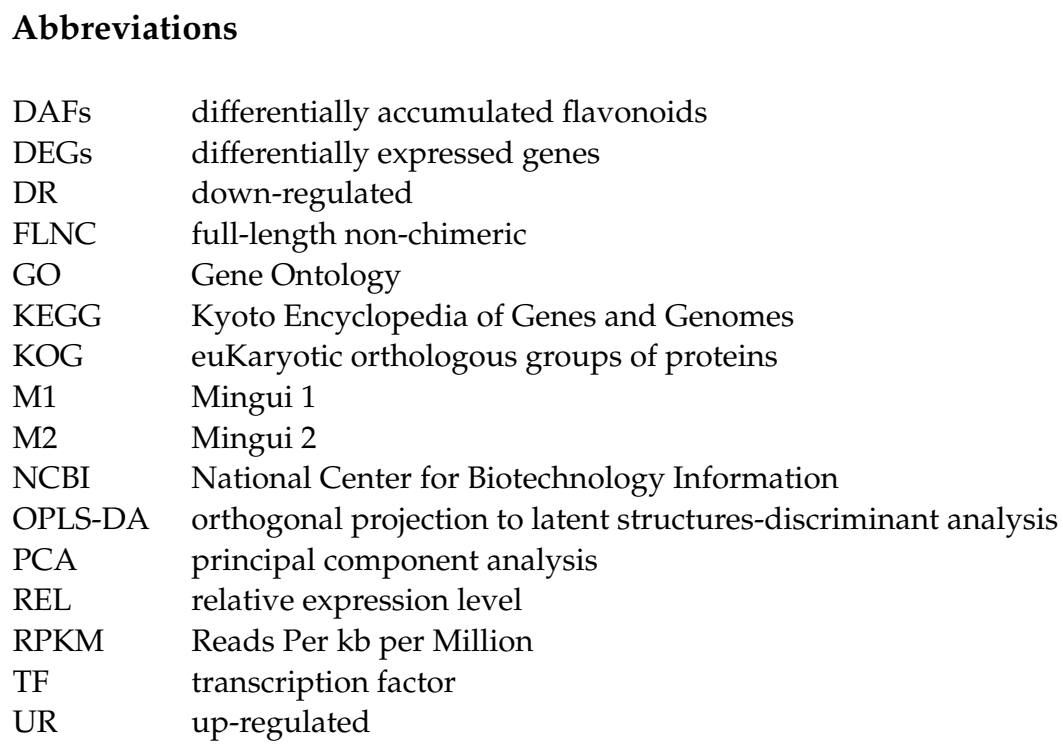

\section{References}

1. Upton, R. American Herbal Pharmacopoeia and Therapeutic Compendium: Dang Gui Root-Angelica sinensis (Oliv.); American Herbal Pharmacopoeia: Scotts Valley, CA, USA, 2003; pp. 1-41.

2. Zhang, H.Y.; Bi, W.G.; Yu, Y.; Liao, W.B. Angelica sinensis (Oliv.) Diels in China: Distribution, cultivation, utilization and variation. Genet. Resour. Crop Evol. 2012, 59, 607-613. [CrossRef]

3. $\quad$ Li, M.F.; Liu, X.Z.; Wei, J.H.; Zhang, Z.; Chen, S.J.; Liu, Z.H.; Xing, H. Selection of high altitude planting area of Angelica sinensis based on biomass, bioactive compounds accumulation and antioxidant capacity. Chin. Tradit. Herbal Drugs 2020, 51, 474-481.

4. Chinese Pharmacopoeia Commission. Pharmacopoeia of the People's Republic of China (2015 Version) Volume I; Chinese Medical Science Press: Beijing, China, 2020; p. 139.

5. Wei, W.L.; Zeng, R.; Gu, C.M.; Qu, Y.; Huang, L.F. Angelica sinensis in China-A review of botanical profile, ethnopharmacology, phytochemistry and chemical analysis. J. Ethnopharmacol. 2016, 190, 116-141. [CrossRef]

6. Han, Y.; Chen, Y.; Zhang, Q.; Liu, B.W.; Li, Y.; Xu, Y.H.; Zhao, Y.H. Overview of therapeutic potentiality of Angelica sinensis for ischemic stroke. Phytomedicine 2021, 90, 153652. [CrossRef]

7. Wang, B.H.; Ou Yang, J.P. Pharmacological actions of sodium ferulate in cardiovascular system. Cardiovasc. Drug. Rev. 2005, 23, 161-172. [CrossRef]

8. Ma, J.P.; Gu, Z.B.; Jin, L.; Li, Y.D. Phytochemical progress made in investigations of Angelica sinensis (Oliv.) Diels. Chin. J. Nat. Med. 2015, 13, 241-249. [CrossRef]

9. Xu, X.Q.; Zhu, T.T.; Shi, T.T.; Chen, J.; Jin, L. Quality suitability regionalization analysis of Angelica sinensis in Gansu, China. PLoS ONE 2020, 15, 0243750. [CrossRef]

10. Lin, H.M.; Wu, Y.A.; Cao, Z.F.; Lv, S.L.; Mao, X.J. Influence of sun shade cultivation on premature bolting in Angelica sinensis and growth environment factors. Chin. J. Exp. Tradit. Med. Form. 2010, 16, 79-83.

11. Liu, B.; Yao, B.Y.; Qian, C.; Wu, Z.J. Effect on different irrigation treatments on growth, yield and quality of Angelica sinensis. Mod. Agr. Sci. Technol. 2013, 2, 77-82.

12. Xiang, H.; Li, Y.; Jin, Y. Effects of light intensity on yield and ferulic acid content in Angelica sinensis. J. Trad. Chin. Veter. Med. 2015, 2, 53-54.

13. Li, M.F.; Kang, T.L.; Jin, L.; Wei, J.H. Research progress on bolting and flowering of Angelica sinensis and regulation pathways. Chin. Tradit. Herbal Drugs 2020, 51, 5894-5899.

14. Li, J.; Li, M.L.; Zhu, T.T.; Zhang, X.N.; Li, M.F.; Wei, J.H. Integrated transcriptomics and metabolites at different growth stages reveals the regulation mechanism of bolting and flowering of Angelica sinensis. Plant Biology. 2021, 23, 574-582. [CrossRef]

15. Li, M.F.; Li, J.; Wei, J.H.; Paré, P.W. Transcription controls for early bolting and flowering in Angelica sinensis. Plants 2021, 10, 1931. [CrossRef]

16. Huang, L.Q.; Jin, L. Suitable Technology for Production and Processing of Angelica sinensis; China Pharmaceutical Science and Technology Press: Beijing, China, 2018; pp. 1-14.

17. Li, Y.D. Research on Angelica sinensis (Oliv.) Diels; Science Press: Beijing, China, 2021; pp. 36-45.

18. Jia, J.N. Report on new cultivar breeding of Angelica sinensis (Oliv.) Diels. Chin. Med. Mat. 2005, 28, 649-650.

19. Wang, M.W.; Li, S.; Li, M.; Li, C.Y.; Yang, F.D. Comprehensive evaluation on medicinal quality of Angelica sinensis Radix of different cultivars (strains) by entropy TOPSIS model. Chin. J. Exp. Tradit. Med. Form. 2017, 23, 63-68.

20. Li, S.N.; Wang, Y.Q.; Wang, F.S.; Fan, Q.; Luo, J.; An, P.K.; Zhang, Y.L.; Li, Q.; Peng, T. Metabonomics Analysis on Different Varieties of Angelica sinensis Based on UPLC-Q-TOF-MS. Chin. J. Exp. Tradit. Med. Form. 2020, 26, 138-147. 
21. Ramakrishna, A.; Ravishankar, G.A. Influence of abiotic stress signals on secondary metabolites in plants. Plant. Signal. Behav. 2011, 6, 1720-1731.

22. Riasat, M.; Heidari, B.; Pakniyat, H.; Jafari, A.A. Assessment of variability in secondary metabolites and expected response to genotype selection in fenugreek (Trigonella spp.). Ind. Crop. Prod. 2018, 123, 221-231. [CrossRef]

23. Li, Z.; Rubert-Nason, K.F.; Jamieson, M.A.; Jamieson, M.A.; Raffa, K.F.; Lindroth, R.L. Root Secondary Metabolites in Populus tremuloides: Effects of Simulated Climate Warming, Defoliation, and Genotype. J. Chem. Ecol. 2021, 47, 313-321. [CrossRef]

24. Cushnie, T.P.T.; Lamb, A.J. Antimicrobial activity of flavonoids. Int. J. Antimicrob. Ag. 2005, 26, 343-356. [CrossRef]

25. Forni, C.; Rossi, M.; Borromeo, I.; Feriotto, G.; Platamone, G.; Tabolacci, C.; Mischiati, C.; Beninati, S. Flavonoids: A Myth or a Reality for Cancer Therapy? Molecules 2021, 26, 3583. [CrossRef]

26. Zhuang, H.; Lou, Q.; Liu, H.; Han, H.; Wang, Q.; Tang, Z.; Ma, Y.; Wang, H. Differential Regulation of Anthocyanins in Green and Purple Turnips Revealed by Combined De Novo Transcriptome and Metabolome Analysis. Int. J. Mol. Sci. 2019, 20, 4387. [CrossRef]

27. Chen, C.; Zhou, G.; Chen, J.; Liu, X.; Lu, X.; Chen, H.; Tian, Y. Integrated Metabolome and Transcriptome Analysis Unveils Novel Pathway Involved in the Formation of Yellow Peel in Cucumber. Int. J. Mol. Sci. 2021, 22, 1494. [CrossRef]

28. Ferreyra, M.L.F.; Rius, S.P.; Casati, P. Flavonoids: Biosynthesis, biological functions, and biotechnological applications. Front Plant. Sci. 2012, 3, 222.

29. Saito, K.; Yonekura-Sakakibara, K.; Nakabayashi, R.; Higashi, Y.; Yamazaki, M.; Tohge, T.; Fernie, A.R. The flavonoid biosynthetic pathway in Arabidopsis: Structural and genetic diversity. Plant Physiol. Bioch. 2013, 72, 21-34. [CrossRef]

30. Hirner, A.A.; Seitz, H.U. Isoforms of chalcone synthase in Daucus carota L. and their differential expression in organs from the European wild carrot and in ultraviolet-A-irradiated cell cultures. Planta 2000, 210, 993-998. [CrossRef]

31. Tohge, T.; Nishiyama, Y.; Hirai, M.Y.; Yano, M.; Nakajima, J.; Awazuhara, M.; Inoue, E.; Takahashi, H.; Goodenowe, D.B.; Kitayama, M.; et al. Functional genomics by integrated analysis of metabolome and transcriptome of Arabidopsis plants over-expressing an MYB transcription factor. Plant J. 2005, 42, 218-235. [CrossRef]

32. Helariutta, Y.; Elomaa, P.; Kotilainen, M.; Seppanen, P.; Teeri, T.H. Cloning of cDNA coding for dihydroflavonol-4-reductase (DFR) and characterization of dfr expression in the corollas of Gerbera hybrida var. Regina (Compositae). Plant Mol. Biol. 1993, 22, 183-193. [CrossRef]

33. Linder, T. Taxonomic Distribution of Cytochrome P450 Monooxygenases (CYPs) among the Budding Yeasts (Sub-Phylum Saccharomycotina). Microorganisms 2019, 7, 247. [CrossRef]

34. Ferdausi, A.; Chang, X.M.; Jones, M. Transcriptomic analysis for differential expression of genes involved in secondary metabolite production in Narcissus pseudonarcissus field derived bulb and In Vitro callus. Ind. Crop. Prod. 2021, 168, 113615. [CrossRef]

35. Sunnadeniya, R.; Bean, A.; Brown, M.; Akhavan, N.; Hatlestad, G.; Gonzalez, A.; Symonds, V.V.; Lloyd, A. Tyrosine Hydroxylation in Betalain Pigment Biosynthesis Is Performed by Cytochrome P450 Enzymes in Beets (Beta vulgaris). PLoS ONE 2016, 11, e0149417. [CrossRef]

36. Ahmad, N.; Liu, J.Y.; Xu, T.; Noman, M.; Jameel, A.; Yao, N.; Dong, Y.Y.; Wang, N.; Li, X.W.; Wang, F.W.; et al. Overexpression of a Novel Cytochrome P450 Promotes Flavonoid Biosynthesis and Osmotic Stress Tolerance in Transgenic Arabidopsis. Genes 2019, 10, 756. [CrossRef]

37. Yu, J.Y.; Hu, F.; Dossa, K.; Wang, Z.K.; Ke, T. Genome-wide analysis of UDP-glycosyltransferase super family in Brassica rapa and Brassica oleracea reveals its evolutionary history and functional characterization. BMC Genom. 2017, 18, 474. [CrossRef]

38. Chen, D.; Chen, R.; Wang, R.; Li, J.; Xie, K.; Bian, C.; Sun, L.; Zhang, X.; Liu, J.; Yang, L.; et al. Probing the Catalytic Promiscuity of a Regio- and Stereospecific C-Glycosyltransferase from Mangifera indica. Angew. Chem. Int. Ed. Engl. 2015, 54, 12678-12682. [CrossRef]

39. Griesser, M.; Vitzthum, F.; Fink, B.; Bellido, M.L.; Raasch, C.; Munoz-Blanco, J.; Schwab, W. Multi-substrate flavonol Oglucosyltransferases from strawberry (Fragaria $\times$ ananassa) achene and receptacle. J. Exp. Bot. 2008, 59, 2611-2625. [CrossRef]

40. Jones, P.; Messner, B.; Nakajima, J.; Schaffner, A.R.; Saito, K. UGT73C6 and UGT78D1, glycosyltransferases involved in flavonol glycoside biosynthesis in Arabidopsis thaliana. J. Biol. Chem. 2003, 278, 43910-43918. [CrossRef]

41. Lim, E.K.; Ashford, D.A.; Hou, B.; Jackson, R.G.; Bowles, D.J. Arabidopsis glycosyltransferases as biocatalysts in fermentation for regioselective synthesis of diverse quercetin glucosides. Biotechnol. Bioeng. 2004, 87, 623-631. [CrossRef]

42. Montefiori, M.; Espley, R.V.; Stevenson, D.; Cooney, J.; Datson, P.M.; Saiz, A.; Atkinson, R.G.; Hellens, R.P.; Allan, A.C. Identification and characterisation of F3GT1 and F3GGT1, two glycosyltransferases responsible for anthocyanin biosynthesis in red-fleshed kiwifruit (Actinidia chinensis). Plant J. 2011, 65, 106-118. [CrossRef]

43. Suzuki, H.; Nakayama, T.; Yonekura-Sakakibara, K.; Fukui, Y.; Nakamura, N.; Yamaguchi, M.A.; Tanaka, Y.; Kusumi, T.; Nishino, T. cDNA cloning, heterologous expressions, and functional characterization of malonyl-coenzyme a:anthocyanidin 3-o-glucoside-6"-o-malonyltransferase from dahlia flowers. Plant Physiol. 2002, 130, 2142-2151. [CrossRef]

44. Suzuki, H.; Sawada, S.; Watanabe, K.; Nagae, S.; Yamaguchi, M.A.; Nakayama, T.; Nishino, T. Identification and characterization of a novel anthocyanin malonyltransferase from scarlet sage (Salvia splendens) flowers: An enzyme that is phylogenetically separated from other anthocyanin acyltransferases. Plant J. 2004, 38, 994-1003. [CrossRef]

45. Ambawat, S.; Sharma, P.; Yadav, N.R.; Yadav, R.C. MYB transcription factor genes as regulators for plant responses: An overview. Physiol. Mol. Biol. Plants 2013, 19, 307-321. [CrossRef] 
46. Nakatsuka, T.; Saito, M.; Yamada, E.; Fujita, K.; Kakizaki, Y.; Nishihara, M. Isolation and characterization of GtMYBP3 and GtMYBP4, orthologues of R2R3-MYB transcription factors that regulate early flavonoid biosynthesis, in gentian flowers. J. Exp. Bot. 2012, 63, 6505-6517. [CrossRef]

47. Pierantoni, L.; Dondini, L.; Franceschi, P.D.; Musacchi, S.; Winkel, B.S.J.; Sansavini, S. Mapping of an anthocyanin-regulating MYB transcription factor and its expression in red and green pear, Pyrus communis. Plant Physiol. Biochem. 2010, 48, 1020-1026. [CrossRef]

48. Chen, Y.; Yang, X.; He, K.; Liu, M.; Li, J.; Gao, Z.; Lin, Z.; Zhang, Y.; Wang, X.; Qiu, X.; et al. The MYB transcription factor superfamily of Arabidopsis: Expression analysis and phylogenetic comparison with the rice MYB family. Plant Mol. Biol. 2006, 60, 107-124.

49. Borevitz, J.O.; Xia, Y.; Blount, J.; Dixon, R.A.; Lamb, C. Activation tagging identifies a conserved MYB regulator of phenylpropanoid biosynthesis. Plant Cell 2000, 12, 2383-2393. [CrossRef]

50. Zimmermann, I.M.; Heim, M.A.; Weisshaar, B.; Uhrig, J.F. Comprehensive identification of Arabidopsis thaliana MYB transcription factors interacting with R/B-like BHLH proteins. Plant J. 2004, 40, 22-34. [CrossRef]

51. Ma, M.L.; Karsani, S.A.; Mohajer, S.; Malek, S.N.A. Phytochemical constituents, nutritional values, phenolics, flavonols, flavonoids, antioxidant and cytotoxicity studies on Phaleria macrocarpa (Scheff.) Boerl fruits. BMC. Complement. Altern. Med. 2014, 14, 11871198.

52. Lv, M.; Su, H.Y.; Li, M.L.; Yang, D.L.; Yao, R.Y.; Li, M.F.; Wei, J.H. Effect of UV-B radiation on growth, flavonoid and podophyllotoxin accumulation, and related gene expression in sinopodophyllum hexandrum. Plant Biol. 2021, 23, 202-209. [CrossRef]

53. Meng, X.C.; Zhang, Y.J.; Wang, X.Q. Content changes of anthocyanin, reducing sugar and soluble protein during the flower development of Petunia hybrid. J. S. China Norm. Univ. 2001, 2, 96-99.

54. Gordon, S.P.; Tseng, E.; Salamov, A.; Zhang, J.; Meng, X.; Zhao, Z.; Kang, D.; Underwood, J.; Grigoriev, I.V.; Figueroa, M.; et al. Widespread polycistronic transcripts in fungi revealed by single-molecule mRNA sequencing. PLoS ONE 2015, 10, e0132628.

55. Li, M.F.; Sun, P.; Kang, T.L.; Xing, H.; Yang, D.L.; Zhang, J.L.; Paré, P.W. Mapping podophyllotoxin biosynthesis and growth-related transcripts with high elevation in Sinopodophyllum hexandrum. Ind. Crop. Prod. 2018, 124, 510-518. [CrossRef]

56. Grabherr, M.G.; Haas, B.J.; Yassour, M.; Levin, J.Z.; Thompson, D.A.; Amit, I.; Adiconis, X.; Fan, L.; Raychowdhury, R.; Zeng, Q.D.; et al. Full-length transcriptome assembly from RNA-Seq data without a reference genome. Nat. Biotechnol. 2011, 29, 644-652. [CrossRef]

57. Mortazavi, A.; Williams, B.A.; McCue, K.; Schaeffer, L.; Wold, B. Mapping and quantifying mammalian transcriptomes by RNA-Seq. Nat. Methods 2008, 5, 621-628. [CrossRef]

58. Love, M.I.; Huber, W.; Anders, S. Moderated estimation of fold change and dispersion for RNAseq data with DESeq2. Genome Biol. 2014, 15, 550. [CrossRef]

59. Robinson, M.D.; McCarthy, D.J.; Smyth, G.K. EdgeR: A Bioconductor package for differential expression analysis of digital gene expression data. Bioinformatics 2010, 26, 139-140. [CrossRef]

60. Willems, E.; Leyns, L.; Vandesompele, J. Standardization of real-time PCR gene expression data from independent biological replicates. Anal. Biochem. 2008, 379, 127-129. [CrossRef] 